\title{
O MODELO DE CICLO ECONÔMICO DE KALECKI
}

\author{
Mario Luiz Possas* \\ Paulo E. A. Baltar*
}

\section{RESUM0}

Este artigo apresenta uma análise formal dos modelos de ciclo econômico desenvolvidos por: Kalecki nas duas versões mais conhecidas, de 1933 e 1954. Após um breve comentārio sobre o conteúdo eco nômico destes modelos, eles são expostos e interpretados matematicamente como solução de equaç̄es a diferenças finitas de 2 ạ ordem com coeficientes constantes. Para facilitar o leitor interessado na comparação com as formulações teóricas originais deste autor manti veram-se as mesmas hipóteses, terminologia e notação. Segue-se uma sucinta análise dos efeitos de distintas taxas de crescimento setoriais sobre a distribuição macroeconômica da renda, que não são tratados nos modelos agregados de ciclo, com base no esquema de 3 setores de Kalecki. Conclui-se com observações sobre as relações en tre tendēncia e ciclo e as perspectivas de aprofundamento teóricc nesse campo.

\section{ABSTRACT}

This article presents a rormal analysis of the business cicle models put forward by Kalecki in the two most familiar versions of 1933 and 1954. After a short comment on the economic content of these models, they are expounded and interpreted mathematically as a solution of 2 nd order difference equations with constant coefficients. With a view to making it easier for the reader a comparison with Kalecki's original theoretical formulations, his assumptions, terminology and notation have been kept throughout. A brief analysis follows dealing with the effects of different sectoral growth rates on the macroeconomic distribution of income, which are not:

* Do Departamento de Economia da UNICARP--Lniversidade Estadual de Campinas. 
focused in aggregate cycle models, based on Kalecki's 3-sector scheme. Concluding observations are made on the relations between trend and cycle and on the perspectives of further theoretical effort in this field. 


\section{INTRODUCAOO}

Como em outras contribuições teóricas, Kalecki expôs sua teoria do ciclo econômico em sucessivos modelos, visando seu progressivo aperfeiçoamento. Duas destas versões, que se tornarammais co nhecidas, serāo objeto de uma exposiçāo e interpretaçāo formais no presente artigo(1): a contida no ensaio "Esboço de uma teoria do cí clo econômico", publicado em 1933, e a constante dos ültimos capítulos do seu livro Teria da Dinâmica Econômica, de 1954. Existe uma terceira versāo, um pouco menos conhecida (ver Kalecki, 1968b), onde procura integrar a tendência com o ciclo econômico, e que deixaremos de lado por motivos expostos adiante.

o interesse em desenvolver um tratamento matemático destes mo delos decorre, a nosso ver, da natureza do problema econômico em questāo, e nāo de uma possível presunçāo genérica de que métodos matemáticos sāo, em regra, mais adequados em Economia. A família de modelos macroeconômicos de crescimento e ciclo desenvolvidos como aplicaçāo dinâmica do princípio da demanda efetiva (às vezes chama dos de "pós-Keynesianos"), à qual pertencem os de Kalecki, baseiase na interaçāo entre a determinação do nível de renda pelo investimento e pelo consumo (agregado ou nāo), de um lado - o efeito de tipo "multiplicador" - e a determinação do nível de investimento a partir do nível e variação da renda (ou de algum prøxy), de outro lado - o efeito de tipo "acelerador", ou mais amplamente de "ajustamento do estoque de capital". Esta interaçāo pode produzir trajetórias muito diferentes em funçāo dos parâmetros estruturaise de comportamento introduzidos no modelo, incluindo-se ai as defasa-

(1) A parte matemática deste artigo constituia originalmente apêndice de outro trabalho nosso (Possas e Baltar, 1981), não tendo sido publicado naquela oportunidade por falta de espaso. 
gens, mas seu resultado é muito pouco intuitivo, exigindo uma ela boração formal considerável. Provavelmente por isso, modelos estru turalmente idênticos, não apenas a nível formal mas teórico, tên sur gido na literatura artificialmente separados em modelos ora de "cres cimento", ora de "ciclo", sem que os motivos para tanto sejam satisfatoriamente justificados devido à ausência de uma análise mais rigorosa das implicações das hipóteses formuladas ${ }^{(2)}$.

Apesar de expor seus modelos com um arcabouço matemático, onde não só cada variável, mas cada parâmetro é definido teoricamen te, Kalecki fornece apenas uma explicacão intuitiva das caracterís ticas da trajetória gerada e sua relação com a forma das funções e os valores dos parâmetros. Assim, tendo em conta o crescente re conhecimento da importância da contribuição deste autor para a aná lise da dinâmica econômica - ao lado de nossa conviç̧ão, exposta em outro artigo já citado, de que sua teoria do ciclo econômico "puro" fornece a explicação básica mais convincente para o mecanismo das flutuações endógenas das economias capitalistas -, parece justificar-se um esforço de formalização adicional.

Além disso, as tentativas anteriores de que temos notícia na literatura (3) não são inteiramente satisfatórias, por dois motivos principais: primeiro, porque o modelo é interpretado como uma equação mista, diferencial e a diferenças finitas, em contraste com a formulação original de Kalecki, que opera unicamente com períodos de tempo discretos. Estes são conceitualmente mais adequados ao tratamento de variáveis econômicas, tanto nas funções de comportamento de agentes econõmicos (investimento e consumo) e respectivas defasagens como no processo de determinação ou ajustamento de variāveis agregadas (níveis de salārios, lucros e renda global, no mo delo simplificado). Em segundo lugar, porque são introduzidas modị. ficações desnecessārias na notação e nas prōprias equações, dificultando a comparação e interpretação dos resultados à luz das prẹ missas teóricas do autor. Nossa intenção é a de contribuir para prẹ

(2) Ver a respeito Pasinetti (1974), cap. 3.

(3) Veja-se Allen (1960), cap. 8, e as referēncias ai contidas. 
encher esta lacuna, oferecendo uma alternativa que envolve exclusi vamente equações a diferenças finitas e que sāo derivadas diretamente das proposições de Kalecki(4), com o mínimo de modificações e hipóteses simplificadoras.

Os itens 2 e 3 a seguir expõem respectivamente os modelos de ciclo de 1933 e 1954. O modelo de 1968, que tenta integrar alguns elementos anteriormente atribuídos à tendência no próprio mecanismo endógeno do ciclo, nāo nos parece suficientemente relevante para merecer um tratamento à parte. A tentativa que nele é feita de tornar enđógenos os efeitos do progresso técnico através de altera ções na rentabilidade esperada dos investimentos devido a variações de produtividade pouco acrescenta ao modelo de 1954, não só porque capta apenas um aspecto parcial destes efeitos, mas principalmente porque equivale tão_somente, do ponto de vista analitico, a explicitar uma das causas de variações dos parâmetros da equação do ciclo. A tendência temporal de crescimento continua sendo explí cada exogenamente pelos "fatores de desenvolvimento"; nāo há de fa to uma integraçāo entre ciclo e tendēncia, no sentido de um princi pio explicativo único, pois ela é impossivel neste tipo de mode $10^{(5)}$. Como modelo teórico, portanto, o de 1954 é preferível, por identificar de modo mais claro e simples o mecanismo do ciclo, separadamente dos fatores explicativos da tendência. Por outro lado, este modelo parece-nos também preferível ao de 1933, ao contrário da opiniāo de outros comentaristas (6). Isto por trēs razões: 19) o efeito da rentabilidade passada do capital, como proxy da rentabilidade esperada, sobre o novo investimento é representado mais ade quadamente por suas variações e não pelo seu nível; 2\%) é inseri-

(4) Incluindo os supostos em geral pouco realistas de funça investimento linear e coeficientes constantes. Note-se, porém, que os modelos de Kalecki não se destinam primordialmente a uma descricão fiel do comportamento das va riäveis ao longo de um ciclo económico real, mas a explicitar os seus mecanismos essenciais de funcionamento. Trata-se portanto de modelos teóricos, e não estatísticos (veja-se a propósito Kalecki, 1954, Prefácio), de forma que, se eles cumprem adequadamente sua finalidade, o relaxamento destas e outras hipóteses simplificadoras apenas modifica os resultados, sem alterar suas características bäsicas.

(5) Como mostrou Pasinetti (1974), cap. 3, nota 17.

(6) Por exemplo, Allen (1960), p. 303. Veja-se opinião oposta do autor em Kale cki (1969), Prefäcio, p.1. 
do um termo independente destinado a captar os efeitos de longo pra zo da introduçāo de progresso técnico, inovações e expansāo dos mex cados, responsável pela existência de uma tendência superposta ao ciclo puro; 30) principalmente, sāo introduzidos fatores de risco microeconômicos afetando a decisāo de reinvestir os lucros das empresas, ligados às condições de endividamento e de mercado( 7 ).

No item 4 apresentamos alguns resultados relacionando tendências de variaçāo na composiçāo da produçāo e da demanda final com alterações macroeconōmicas na distribuiçāo funcional da renda, a partir do esquema de 3 macrossetores verticalmente integrados de Ka lecki (1968a). Em sua teoria da determinaçāo da renda, a distribuiçāo entre lucros e salārios cumpre um papel paramétrico, da mes ma forma que na teoria do ciclo, uma vez que seus fatores explicativos estāo localizados no processo microeconômico de formação de preços e não a nivel agregado. Mas isto obviamente nāo significa que a distribuição permaneça constante em termos macroeconômicos, pois ela pode variar com a composição do produto e esta certamente se altera no transcurso do ciclo; nem, ao inverso, impede que ela possa afetar alguns parâmetros do ciclo. Embora a hipótese de cons tância dos coeficientes nos modelos de ciclo obscureça estes efeitos dināmicos de mudanças na distribuição, a forma pela qual, ao inverso, a distribuiçāo macroeconômica tende a ser afetada por dis tintas taxas de crescimento setoriais pode ser facilmente analisada com base no modelo de 3 setores proposto por Kalecki. Nossa abordagem é muito simplificada, mas permite sugerir uma fraçāo das potencialidades ainda inexploradas deste esquema analitico.

2. 0 MODELO DE CICLO DE KALECKI, 1 ą VERSÃO (1933)

- Definições: $\mathrm{P}=$ lucros; $\mathrm{C}=$ consumo dos capitalistas; $\mathrm{B}=$ = parte constante do consumo dos capitalistas.

$I=$ investimento bruto (encomendas); $A=$ produçāo e entrega (8) de bens de investimento, sendo $A_{t}=I_{t}-\theta^{\prime}$ onde $\theta$ é o período mé-

(7) Tratamos destes aspectos pormenorizadamente no artigo citado, pp. 140 e seg.

(8) Desprezando-se o intervalo de tempo entre ambas. 
dio de construção e instalaçāo dos equipamentos e $t$ um período de tempo discreto $(t=1,2 \ldots n) ; K=$ capital fixo (no início do perio do) .

2.1. APRESENTACAOO DO MODELO

Tem-se:

$$
\begin{aligned}
& P_{t}=c_{t}+A_{t} \\
& c_{t}=B+\lambda P_{t}
\end{aligned}
$$

sendo $\lambda$ constante a curto prazo.

De (1) e (2):

$$
P_{t}=\frac{B+A_{t}}{1-\lambda}
$$

Supõe-se que as decisões de investir são dadas pela função:

$$
\frac{I_{t}}{K_{t}}=f\left(\frac{P_{t}}{K_{t}}\right)
$$

onde $\frac{\mathrm{P}}{\mathrm{K}^{\prime}}$, a rentabilidade real do capital existente, é tomada como es timativa da rentabilidade esperada do novo capital.

De (3), tem-se $\frac{P}{K}$ proporcional a $\frac{B+A}{K}$, donde (4) pode ser es crita como

$$
\frac{I_{t}}{K_{t}}=\varnothing\left(\frac{B+A_{t}}{K_{t}}\right)
$$

Na suposiçāo mais simples de uma função linear:

$$
\begin{aligned}
& \frac{I_{t}}{K_{t}}=m \frac{B+A_{t}}{K_{t}}-n, \text { ou }: \\
& I_{t}=m\left(B+A_{t}\right)-n K_{t}
\end{aligned}
$$

Deve-se ter $m>0$, sendo $\varnothing$ uma função crescente, e $n>0$ para que o modelo comporte a ocorrência de ciclos econômicos (como se verá adiante). 
Em cada período de tempo $t$ se tem, por definição:

$\Delta \mathrm{K}_{\mathrm{t}}=\mathrm{K}_{\mathrm{t}+1}-\mathrm{K}_{\mathrm{t}}=\mathrm{A}_{\mathrm{t}}-\mathrm{U}(7)$, onde $\mathrm{U}$ representa a reposição de capital, cujas flutuações se supõe desprezíveis.

Lembrando que $I_{t}=A_{t+\theta}$ e definindo $\theta=1$ lo período $\theta$ tomado como unidade de medida do transcurso do tempol, pode-se substituir (7) em (6), obtendo-se:

$$
k_{t+2}-(m+1) k_{t+1}+(m+n) k_{t}=m B+(m-1) v
$$

A expressão acima é uma equação a diferenças finitas de 2 å or dem, com coeficientes constantes e termo constante, do tipo

$$
y_{t+2}+a_{1} Y_{t+1}+a_{2} y_{t}=c .
$$

A solução da equação reduzida (homogênea) correspondente (abstrain do o termo constante, $c=0$ ) é do tipo $y_{t}=k x^{t}$, sendo $k$ e $x$ coef $\underline{\text {. }}$ cientes a determinar. Substituindo-a na equação homogênea obtém-se, após simplificação, $x^{2}+a_{1} x+a_{2}=0$. As características das raízes $\left(x_{1}, x_{2}\right)$ desta equação definirão o comportamento da variável y no tempo. Assim, só a obtenção de raízes complexas conjugadas ( $x_{1}$ ' $\left.x_{2}=\alpha \pm \beta i\right)$, sob a condição $a_{1}{ }^{2}<4 a_{2}$, garante a ocorrência de flutuações cíclicas neste modelo. A solução homogênea (que só abrange as flutuações) será então da forma $y_{t}=a r^{t} \cos (\omega t+\varepsilon)$, sendo a e $\varepsilon$ arbitrárias (dependendo somente de condições iniciais), e:

$$
\omega=\cos ^{-1}\left(\frac{\alpha}{\sqrt{a_{2}}}\right), r=\sqrt{a_{2}}
$$

No modelo em questão, a condição de ocorrência de flutuações cíclicas se traduz da seguinte forma:

$a_{1}=-(m+1) ; a_{2}=m+n ;$ logo, a condição de $a_{1}{ }^{2}<4 a_{2}$, se expressa como

$$
\begin{gathered}
(m+1)^{2}<4(m+n) ; \text { ou } m^{2}+2 m+1<4 m+4 n \text {, donde: } \\
(m-1)^{2}<4 n \quad \text { (9) }
\end{gathered}
$$


Da condição (9) deduz-se diretamente $\underline{n>0}$.

Finalmente, os coeficientes $\omega$ e $r$ terão os valores:

$$
\omega=\cos ^{-1}\left(\frac{m+1}{2 \sqrt{m+n}}\right) \quad \text { e } \quad r=\sqrt{m+n},
$$

e as flutuações do estoque de capital $K$ se farão segundo a função

$$
K_{t}=a r^{t} \cos (\omega t+\varepsilon)+\bar{K},
$$

onde $a=\frac{x_{0}}{\cos \varepsilon}$ e $\varepsilon$ (fase) é arbitrário, e o nível de "equilíbrio" do capital $\bar{K}=\frac{m}{n} B+\frac{m-1}{n} U$.

Portanto, as flutuaçōes de $\mathrm{K}$ terão período constante igual a $2 \pi / \omega$ unidades de tempo (sendo $\omega$ em radianos) e amplitude a $r^{t}$, que pode ser:

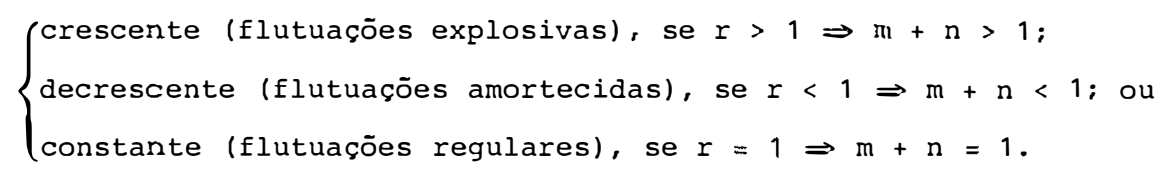

As demais variáveis agregadas (renda total, lucros, investimento) registrarão o mesmo comportamento (período, amplitude, etc.) - por exemplo, a equação do investimento será

$$
A_{t+2}-(m+1) A_{t+1}+(m+n) A_{t}=n U ;
$$

a única diferença é que sua fase será $\varepsilon+\delta$, representando um retardo de $\delta$ das flutuações do estoque de capital em relação a estas variáveis (a defasagem $\delta$ é tal que

$$
\left.\operatorname{tg} \delta=\frac{\sqrt{4 n-(m-1)^{2}}}{m-1}\right)
$$

(9) Para o cálculo deste retardo seguiu-se o mesmo procedimento de Pasinetti (1974), cap. 3, apêndice. 


\subsection{INTERPRETACAOO}

Dada a equação caracteristica $x^{2}+a_{1} x+a_{2}=0$ correspondente à equação a diferenças finitas que expressa 0 estoque de capital como variável temporal - ver item 1, equação (8) - e sendo os coeficientes

$$
a_{1}=-(m+1) \quad \text { e } \quad a_{2}=(m+n)
$$

a relação entre as raỉes $x_{1} e x_{2}$, que definem a trajetōria no tem po da variável, será dada por:

$$
\begin{aligned}
& x_{1}+x_{2}=-a_{1}=m+1 \quad \text { (1), } \\
& x_{1} \cdot x_{2}=a_{2}=m+n
\end{aligned}
$$

Se $a_{1}{ }^{2} \geq 4 a_{2}$ ou $(m-1)^{2} \geq 4 n$, as raizes $x_{1}$ e $x_{2}$ serão reais.

Como $m$ e $n$ são por hipōtese positivos, resulta de (2) que $x_{1}$ $x_{2}$ terão o mesmo sinal, e de (1) que $x_{1}$ e $x_{2}$ são ambos positivos.

Além disso, se supusermos que $x_{1}$ é a raiz dominante (de maior valor absolutol, a possibilidade de que simultaneamente $x_{1} \geq 1 \mathrm{e}$ $\mathrm{x}_{2}<1$ está excluída de início, dado que ela implicaria

$$
\begin{aligned}
& \left(\mathrm{x}_{1}-1\right)\left(\mathrm{x}_{2}-1\right) \leqq 0, \\
& \mathrm{x}_{1} \cdot \mathrm{x}_{2}-\left(\mathrm{x}_{1}+\mathrm{x}_{2}\right)+1 \leqq 0 ; \text { de }(1) \text { e }(2), \\
& \mathrm{m}+\mathrm{n}-(\mathrm{m}+1)+1 \leqq 0, \text { ou } \\
& \mathrm{n} \leq 0, \text { o que por hipótese é impossivel. }
\end{aligned}
$$

Logo, restam para $x_{1}$ e $x_{2}$ apenas as possibilidades de que $x_{1}$ e $x_{2}<1$ ou $x_{1}$ e $x_{2}>1$, correspondentes, respectivamente, a um comportamento temporal amortecido ou explosivo da variável, e que podem ser substituidas pelas condiçōes $x_{1} \cdot x_{2}<1$ ou $x_{1} \cdot x_{2}>1$, isto é: 
I) $\left\{\begin{array}{l}m+n<1 \Longleftrightarrow \text { amortecimento; } \\ m+n>1 \Longleftrightarrow \text { crescimento explosivo. }\end{array}\right.$

A condição de flutuaçōes cíclicas está associada a $x_{1,2}$ complexas conjugadas, o que implica $(\mathrm{m}-1)^{2}<4 \mathrm{n}$.

Temos então, no que se refere à ocorrência de ciclos:

II $\left\{\begin{array}{l}n>\left(\frac{1-m}{2}\right)^{2} \Longleftrightarrow \text { flutuações cíclicas; } \\ n \leqq\left(\frac{1-m}{2}\right)^{2} \Longleftrightarrow \text { expansão sem flutuação. }\end{array}\right.$

Os valores dos coeficientes que correspondem à combinação destas possibilidades estão representados no gráfico a seguir.

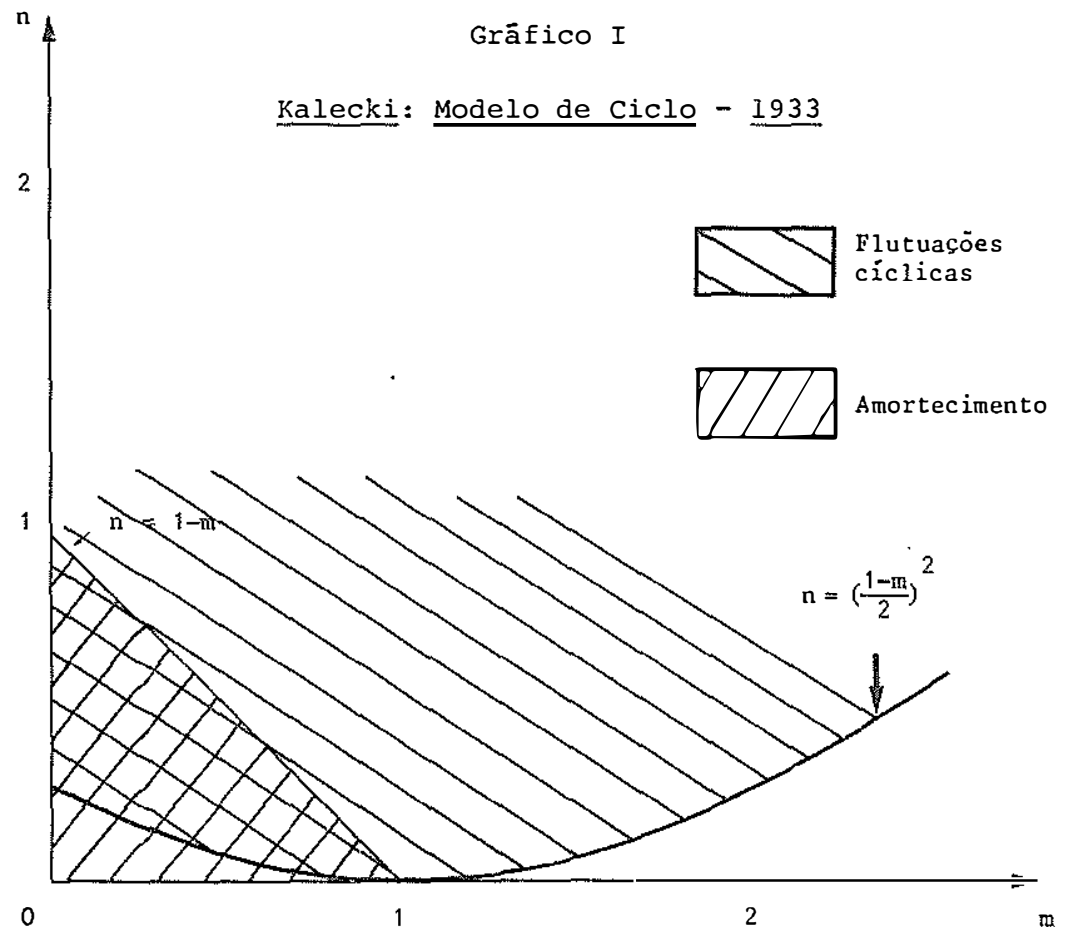


3. 0 MODELO DE CICLO DE KALECKI, 2ạ VERSÃO (1954)

- Definições: P = lucros brutos; $C$ = consumo dos capitalistas; $\mathrm{A}=$ parte constante do consumo dos capitalistas; $\mathbf{S}$ = poupança bruta (no caso geral, igual ao investimento bruto mais saldo da ba lança comercial e déficit orçamentáriol.

$O=$ produto bruto; $Y=$ renda bruta do setor privado; $E=$ impostos indiretos; $\mathrm{B}=$ parcela constante da participacão dos salários e ordenados na renda; $\alpha$ = participação dos salários e ordenados na renda.

$D=$ decisões de investimento bruto em capital fixo; $F$ = inves timento bruto em capital $f i x o$, sendo $F_{t+\tau}=D_{t} ; J=$ investimento em estoques; $I=$ investimento bruto total; $\delta=$ depreciação do capital fixo; $K=$ estoque de capital fixo.

3.1. APRESENTACÁO DO MODELO

Tem-se:

$$
P_{t}=C_{t}+I_{t}
$$

(fazendo implicitamente $I_{t}=s_{t}$ );

$$
c_{t}=q P_{t}+A(10)
$$

sendo $0<\mathrm{q}<1$ estável no curto prazo.

De (1) e (2) obtēm-se:

$$
P_{t}=\frac{I_{t}+A}{1-q} \quad \text { (3). }
$$

Tem-se ainda:

$$
\begin{aligned}
Y_{t} & =\frac{P_{t}+B}{1-\alpha} \quad \text { (4). } \\
\text { e } \quad O_{t} & =Y_{t}+E
\end{aligned}
$$

(10) Kalecki supõe uma defasagem $\lambda$ entre C e $P$, que pode ser desprezada, sem pre juízo da validade do modelo, a fim de simplificá-1o. 0 próprio autor sugere isto mais tarde: veja-se Kalecki (1962), p.135. 
De (4) e (5):

$$
O_{t}=\frac{P_{t}+B}{1-\alpha}+E \quad(6)
$$

As decisões de investimento se supõem determinadas por:

$$
D_{t}=a s_{t}+b \frac{\Delta P_{t}}{\Delta t}-c \frac{\Delta K_{t}}{\Delta t}+d \quad \text { (7), }
$$

onde a primeira parcela, a $S_{t}$, reflete a influência da acumulação interna das empresas, as duas seguintes expressam o efeito da rentabilidade esperada na medida em que esta reflete variações no grau de utilização do capital fixo, e a última é uma constante sujeita a variacões no longo prazo (por efeito, entre outros, de inovacōes).

A equação (7) do investimento neste modelo pode ser deduzida da equação análoga do modelo anterior (item 1):

$$
D_{t}=m^{\prime} P_{t}-n K_{t} \quad(6)^{\prime} ;
$$

calculando as primeiras diferenças finitas;

$$
\begin{aligned}
& \frac{\Delta D_{t}}{\Delta t}=m^{\prime} \frac{\Delta P_{t}}{\Delta t}-n \frac{\Delta K_{t}}{\Delta t}, o u: \\
& D_{t}-D_{t-1}=m^{\prime} \frac{\Delta P_{t}}{\Delta t}-n \frac{\Delta K_{t}}{\Delta t} ;
\end{aligned}
$$

Supondo, por simplicidade, que o saldo do comércio exterior e o déficit orçamentário são ambos despreziveis e que os estoques não sofrem variaçōes significativas no ciclo, podemos escrever $\mathrm{F}_{\mathrm{t}}=\mathrm{S}_{\mathrm{t}}$; como, além disso, $F_{t}=D_{t-1}$ (fazendo $\tau=1$ por hipótese), tem-se $D_{t-1}=s_{t}$. Substituindo na equação anterior:

$$
D_{t}=S_{t}+m^{\prime} \frac{\Delta P_{t}}{\Delta t}-n \frac{\Delta K_{t}}{\Delta t} .
$$

Verifica-se que a equação (7) difere da função acima somente em dois aspectos: a presença de um termo constante, que pretende in corporar influências de longo prazo, e a introdução de um coeficien 
te $\underline{a}$, que pode assumir valores distintos de 1, multiplicando a variável $s_{t}$. Isto representa a possibilidade de alterações nas deci sōes de investir de um período para outro, em função de mudanças nas condições de acumulação interna das empresas e nos condicionan tes de longo prazo, mesmo quando não haja modificações nas taxas de lucro efetiva e esperada.

Retornando ao modelo, a equação (7) pode ser reescrita:

$$
F_{t+\tau}=a S_{t}+b \frac{\Delta P_{t}}{\Delta t}-c \frac{\Delta K_{t}}{\Delta t}+d \quad \text { (8). }
$$

Como $\frac{\Delta \mathrm{K}_{\mathrm{t}}}{\Delta \mathrm{t}}=\mathrm{F}_{\mathrm{t}}-\delta$, a equação (8) passa a ser:

$$
F_{t+\tau}=a S_{t}+b \frac{\Delta P_{t}}{\Delta t}-c\left(F_{t}-\delta\right)+d .
$$

Após alguma manipulação algébrica chega-se a:

$$
F_{t+\theta}=\frac{a}{1+c} s_{t}+b^{\prime} \frac{\Delta P_{t}}{\Delta t}+d^{\prime} \text { (9), }
$$

sendo $\theta$ pouco menor que $\tau, b^{\prime}=\frac{b}{1+c}$ e $d^{\prime}=\frac{c \delta+d}{1+c}$.

Supondo-se um comportamento do investimento em estoques relacionado com a taxa de variação da produção,

$$
J_{t+\theta}=e \frac{\Delta O_{t}}{\Delta t} \quad(10)
$$

Finalmente, de (9) e (10) resulta:

$$
I_{t+\theta}=\frac{a}{1+c} S_{t}+b^{\prime} \frac{\Delta P_{t}}{\Delta t}+e \frac{\Delta O_{t}}{\Delta t}+d^{\prime}
$$

As variações dos lucros e do produto podem ser expressas como resultado de variações no investimento, partindo-se das equações (3) e (6):

$$
\Delta \mathrm{P}_{\mathrm{t}}=\frac{\Delta \mathrm{I}_{\mathrm{t}}}{1-\mathrm{q}}
$$




$$
\Delta O_{t}=\frac{\Delta I_{t}}{(1-q)(q-\alpha)}
$$

Substituindo (12) e (13) em (11), e com $I_{t}=S_{t}$, obtém-se:

$$
I_{t+\theta}=\frac{a}{1+c} I_{t}+\mu \frac{\Delta I_{t}}{\Delta t}+d^{\prime} \quad(14) \text {. }
$$

onde $\mu=\frac{1}{1-q}\left(b^{\prime}+\frac{e}{1-\alpha}\right)$.

(14) resulta:

Definindo-se 0 investimento líquido $i_{t}=I_{t}-\delta$, da equação

$$
i_{t+\theta}=\frac{a}{1+c} i_{t}+\mu \frac{\Delta i_{t}}{\Delta t}+g
$$

onde $g$, a tendência do investimento líquido, é dado por

$$
d^{\prime}-\delta\left(1-\frac{a}{1+c}\right) \text {. }
$$

Finalmente, fazendo $\Delta t=\theta=1^{(11)}$, supondo $\Delta i_{t}=i_{t}-i_{t-1}$ e defasando a equação (15) de 1 período, tem-se:

$$
\dot{i}_{t+2}-\left(\mu+\frac{a}{1+c}\right) \dot{i}_{t+1}+\mu \dot{i}_{t}=g \quad(16) .
$$

Esta é uma equação a diferenças finitas de 2 ạ ordem com termo e coeficientes constantes, do mesmo tipo analisado no item anterior. Sua solução completa será então a soma da solução particular $i_{p}$ com a função complementar (solução homogênea) $i_{c}$ :

$$
i_{p}=\frac{g}{1-\frac{a}{1+c}}=\frac{d^{\prime}}{1-\frac{a}{1+c}}-\delta
$$

(11) Equivale a tomar como unidade de tempo o prazo de maturacaão médio do inves timento $\theta$ e considerá-lo igual ao período médio de reação das decisões de investir a mudanças na rentabilidade esperada, $\Delta t$. Esta ültima hipötese è uma simples aproximação, jä que o período de reação é muito impreciso teoricamente. 
e $i_{c}=A_{1} x_{1}{ }^{t}+A_{2} x_{2}{ }^{t}$, onde $x_{1,2}$ são as raízes da equação $x^{2}+a_{1} x$ $+a_{2}=0$ (sendo $a_{1}$ e $a_{2}$ os coeficientes de $i_{t+1}$ e $i_{t}$ na equação (16)).

A condição para que possa haver flutuações cíclicas, como se viu, é $a_{1}{ }^{2}<4 a_{2}$, que dá raízes $x_{1,2}$ complexas conjugadas.

Neste caso,

$$
\begin{aligned}
& \left(\mu+\frac{a}{1+c}\right)^{2}<4 \mu, \text { ou } \\
& \left(\frac{a}{1+c}\right)^{2}+2 \mu\left(\frac{a}{1+c}\right)+\mu^{2}-4 \mu<0 .
\end{aligned}
$$

Esta condição equivale a:

$$
-\mu-2 \sqrt{\mu}<\frac{a}{1+c}<-\mu+2 \sqrt{\mu} .
$$

Como $\frac{a}{1+c}>0$ e $\mu>0$ por hipótese, resulta:

$$
0<\frac{a}{1+c}<-\mu+2 \sqrt{\mu}
$$

Em particular, de (18) obtém-se:

$-\mu+2 \sqrt{\mu}>0$, donde necessariamente $\mu<4$.

Chamando $f(\mu)=-\mu+2 \sqrt{\mu}$, com $\mu \varepsilon(0,4)$, a condição $(18)$ equi vale a:

$$
\frac{a}{1+c}<f(\mu) \text {. }
$$

E facilmente demonstrável que o valor máximo de $f(\mu)$ se obtém para $\mu=1$, onde $f(1)=1$. Assim, a mesma condição se expressa finalmente como:

$$
\frac{a}{1+c}<1
$$

A solução homogênea $i_{c}$ obtida nas condições acima (com flutua ções cíclicas) è então: 


$$
i_{c}=a r^{t}(\omega t+\varepsilon)
$$

onde $a=\frac{i_{0}}{\cos \varepsilon}, \operatorname{com} \varepsilon$ (fase) arbitrário, e

$$
\omega=\cos ^{-1}\left(\frac{\mu+\frac{a}{1+c}}{2 \sqrt{\mu}}\right) \quad \text { e } \quad r=\sqrt{\mu} \text {. }
$$

A solução completa será (somando as expressões 17 e 19):

$$
\dot{i}_{t}=a r^{t} \cos (\omega t+\varepsilon)+\bar{i} \quad(20) \text {. }
$$

Esta equação representa flutuações cíclicas do investimento líquido em torno a um nível de "equilíbrio móvel"

$$
\bar{i}=\frac{d^{\prime}}{1-\frac{a}{1+c}}-\delta,
$$

com período constante de $\frac{2 \pi}{\omega}$ ( $\omega$ em radianos) e amplitude a . r ${ }^{t}$, que pode ser:

$$
\left\{\begin{array}{lll}
\text { crescente } & \text { (flutuaçōes explosivas), } & \text { se } r>1 \Rightarrow \mu>1 \\
\text { decrescente } & \text { (flutuaçōes amortecidas), } & \text { se } r<1 \Rightarrow \mu<1 \\
\text { constante } & \text { (flutuações regulares), } & \text { se } r=1 \Rightarrow \mu=1
\end{array}\right.
$$

As flutuações do nível de renda, dos lucros e do estoque de ca pital terão o mesmo comportamento cíclico (com igual período e ain plitude proporcional), embora as flutuaçōes do estoque de capita $\bar{l}$ apresentem um retardo, $\emptyset$, de modo análogo ao item anterior:

$$
\operatorname{tg} \varnothing=\frac{\sqrt{\mu} \sqrt{4 \mu-\left(\mu+\frac{a}{1+c}\right)^{2}}}{(\sqrt{\mu}-1)\left(\mu+\frac{a}{1+c}\right)} .
$$

\subsection{INT ERPRETACAOO}

Os coeficientes da equação caracteristica são, nesse modelo, 


$$
a_{1}=-\left(\mu+\frac{a}{1+c}\right) \quad \text { e } \quad a_{2}=\mu,
$$

e as suas raízes $x_{1}$ e $x_{2}$ satisfazem as condições:

$$
\begin{aligned}
& x_{1}+x_{2}=\mu+i \frac{a}{+c} \text { (1), } \\
& x_{1}, x_{2}=\mu
\end{aligned}
$$

As raỉzes serão reais se $\left(\mu+\frac{a}{1+c}\right)^{2} \geq 4 \mu$.

Tem-se nesse caso $x_{1}, x_{2}>0$, de (1) e (2), dados $\mu$, a $e \underline{c}$ positivos.

Sendo $x_{1}$ a raiz dominante, as condições $x_{1} \geq 1$ e $x_{2}<1$ não podem ocorrer ao mesmo tempo, pois implicariam

$$
\begin{gathered}
\left(x_{1}-1\right)\left(x_{2}-1\right) \leqq 0, \text { ou } \\
\mu-\left(\mu+\frac{a}{1+c}\right)+1 \leqq 0, \text { ou ainda } \\
\frac{a}{1+c} \geqq 1,
\end{gathered}
$$

o que é impossível por hipótese.

As únicas condições possíveis são, portanto, como no modelo an terior, $x$ e $x_{2}>1$ ou $x_{1}$ e $x_{2}<1$, equivalentes a

$$
x_{1} \cdot x_{2}>1 \text { ou } x_{1} \cdot x_{2}<1,
$$

e que podem ser expressas como:

I) $\left\{\begin{array}{l}\mu<1 \Longleftrightarrow \text { amortecimento: } \\ \mu>1 \Longleftrightarrow \text { crescimento explosivo. }\end{array}\right.$

A existência de ciclos depende de $x_{1}$ e $x_{2}$ serem complexas con jugadas, o que supõe 
ABRIL DE 1983

$$
\left(\mu+\frac{a}{1+c}\right)^{2}<4 \mu
$$

que se reduz, dado $\frac{a}{1+c}>0, a$ :

II) $\left\{\begin{array}{l}\frac{a}{1+c}<-\mu+2 \sqrt{\mu} \Longleftrightarrow \text { flutuações cíclicas; } \\ \frac{a}{1+c} \geq-\mu+2 \sqrt{\mu} \Longleftrightarrow \text { expansão sem flutuação. }\end{array}\right.$

os valores dos parāmetros que dão lugar às distintas combinações de trajetos temporais são representados no gráfico a seguir.

Gräfico II

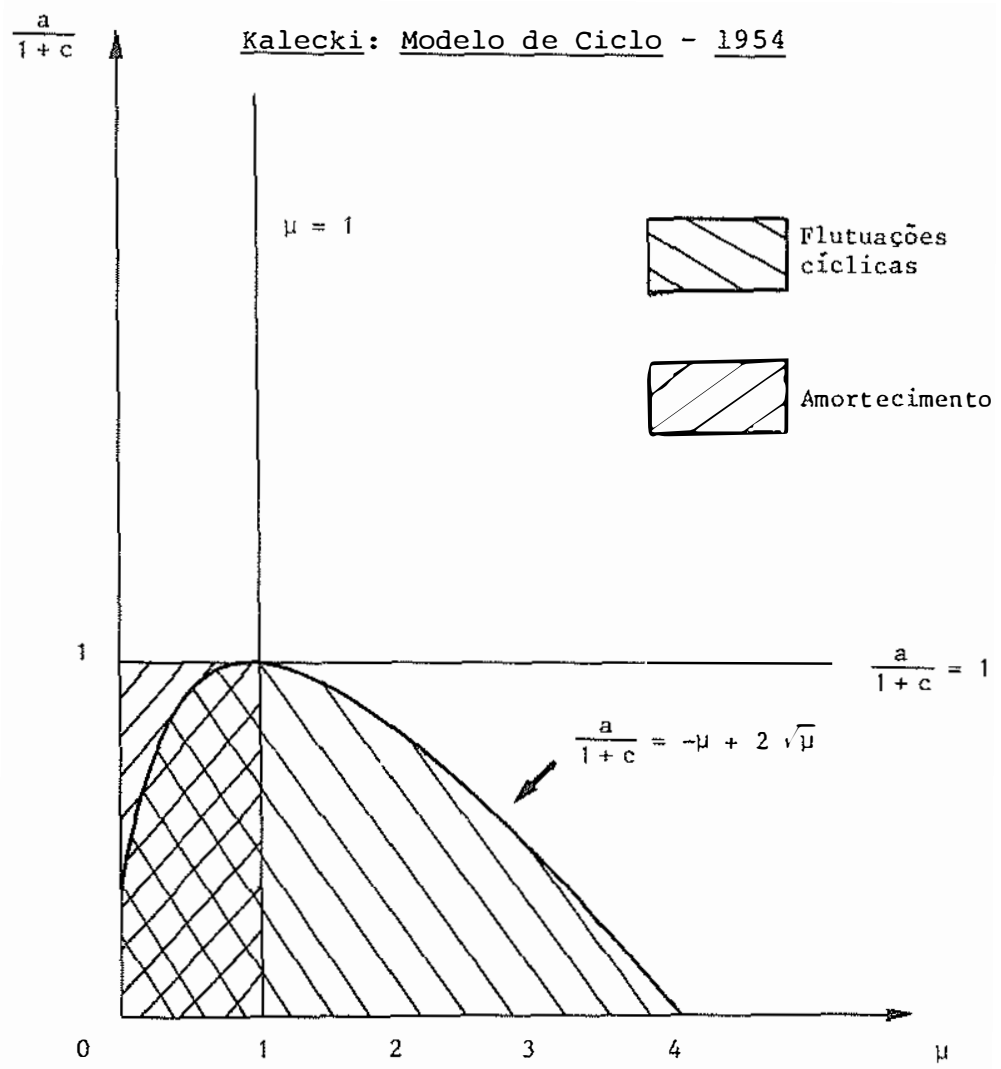


4. DISTRIBUICAOO FUNCIONAL DA RENDA EM KALECKI NUM ENFOQUIE DINAMICO

No que se segue faremos uso da notação empregada por Kalecki no artigo sobre as equações de reprodução (1968a), adotando ainda a hipótese mais geral de que o consumo capitalista é estritamente autônomo (e não uma função determinada do investimento através dos lucros).

- Definições: $Y=$ renda nacional bruta; $I$ = investimento bruto (produção do departamento $I$, ou DI) : $C_{k}=$ consumo dos capitalis tas (produção de DII); $C_{w}=$ consumo dos trabalhadores (produção de DIII); $\quad W=$ salários; $w_{i}$, parāmetros setoriais de distribuição fun cional da renda, $=w_{i} /$ produto de $D_{i}$.

Tem-se:

$\mathrm{Y}=\mathrm{I}+\mathrm{C}_{\mathrm{k}}+\mathrm{C}_{\mathrm{w}}$ (1) e $\mathrm{W}=\mathrm{C}_{\mathrm{w}}$ (2), isto é, os salários são totalmente consumidos (hipótese simplificadora).

Das definições de $w_{i}$ resulta, de (1) e (2):

$$
\mathrm{w}=\frac{\mathrm{w}_{1}{ }^{\mathrm{I}}+\mathrm{w}_{2} \mathrm{c}_{\mathrm{k}}}{1-\mathrm{w}_{3}} \quad \text { (3). }
$$

De (1) e (3) obtém-se:

$$
\frac{W}{Y}=\frac{w_{1} I+w_{2} C_{k}}{\left(1+w_{1}-w_{3}\right) I+\left(1+w_{2}-w_{3}\right) C_{k}}
$$

As condições gerais para que haja ou não uma alteração na dis tribuição funcional macroeconômica da renda, expressa por W/Y, podem ser derivadas da condição geral (12)

$$
d(W / Y) \gtrless 0 \quad(5) \text {. }
$$

(12) A utilizaçāo de diferenciais neste caso não afeta o sentido econōmico das variäveis e parâmetros, uma vez que nāo se pretende deduzir relaçōes funcionais entre os mesmos, e sim apontar as condicões que devem satisfazer associadas a tendéncias de mudança das variäveis. 
Tem-se, no caso mais geral de $I, c_{k}$ e $w_{i}$ variáveis independentes:

$$
\begin{gathered}
d(W / Y)=\frac{\delta(W / Y)}{\delta I} \cdot d I+\frac{\delta}{\delta C_{k}} k+\frac{\delta(W / Y)}{\delta W_{1}} \cdot d w_{1}+ \\
+\frac{\delta(W / Y)}{\delta w_{2}} \cdot d w_{2}+\frac{\delta(W / Y)}{\delta W_{3}} \cdot d w_{3} \cdot
\end{gathered}
$$

Aplicada a (4), a equação acima fornece:

$$
\begin{gathered}
d(X / Y)=\frac{E_{1}}{E_{2}} \text {, onde } \\
E_{1}=\left(w_{1}-w_{2}\right)\left(1-w_{3}\right)\left[C_{k} \cdot d I-I \cdot d C_{k}\right]+\left(I+C_{k}\right) \\
{\left[\left(1-w_{3}\right)\left(I \cdot d w_{1}+c_{k} \cdot d w_{2}\right)+\left(w_{1} \cdot I+w_{2} \cdot c_{k}\right) d w_{3}\right],} \\
E_{2}=\left[\left(1+w_{1}-w_{3}\right) I+\left(1+w_{2}-w_{3}\right) c_{k}\right]^{2} .
\end{gathered}
$$

Sendo necessariamente $E_{2}>0$, a condição (5) se expressa como:

$$
d(W / Y) \geqslant 0 \Longleftrightarrow E_{1} \geqslant 0 \quad \text { (5)'. }
$$

Esta condição expressa a possibilidade de aumentar, manter inalterada ou reduzir a participação dos salários na renda, em funcão do comportamento das variáveis $I$ e $C_{k}$ e dos parāmetros $w_{i}$, aquelas dependentes das decisões (autônomas) dos capitalistas e estes expressando as condições de concorrēncia e poder de negociação entre capitalistas e trabalhadores de cada departamento produtivo. Vejamos algumas possibilidades:

a) Se os parāmetros de distribuição $w_{i}$ permanecem constantes:

$$
\begin{aligned}
& E_{1} \text { reduz-se a } E_{1}=\left(w_{1}-w_{2}\right)\left(1-w_{3}\right)\left[C_{k} \cdot d I-I \cdot d C_{k}\right], \text { e como } \\
& w_{3}<1, \quad(5)^{\prime} \text { passa a ser: }
\end{aligned}
$$




$$
\begin{array}{lll}
d(W / Y)=0 \Longleftrightarrow w_{1}=w_{2} & \text { ou } & \frac{d I}{I}=\frac{d C_{k}}{D_{k}} ; \\
a(W / Y)>0 \Longleftrightarrow w_{1} \gtrless w_{2} & \underline{e} & \frac{d I}{I} \gtrless \frac{d C_{k}}{C_{k}} ;
\end{array}
$$

Portanto, a distribuição de renda agregada só permanecerá cons tante se as taxas de crescimento de DI e DII ou os parāmetros de distribuição respectivos forem iguais. Para que a participação dos salários aumente [ ou diminua ] é necessário (dada a constāncia dos parāmetros de distribuiçãol e suficiente que o departamento (dentre DI e DII) cuja distribuição é mais favorável aos salários cresça mais [ ou menos ] rapidamente.

b) Se os parāmetros de distribuição $\underline{w}_{i}$ são variāveis, pode-se veri ficar a partir da expressão $E_{1}$ que sua influência, em termos gerais, será no sentido de compensar os efeitos sobre a distribuição global da renda exercidos pelo crescimento relativo de DI e DII e conforme os respectivos parāmetros setoriais $\left(w_{1} e w_{2}\right)$. Assim, uma redução geral ou parcial dos parāmetros de distribuição $w_{i}$ devida, por exemplo, a um aumento no grau de monopólio das empresas não acarretará necessariamente a diminuição da participação global dos salārios na renda, desde que DI cresça mais rapidamente que DII(se $w_{1}>w_{2}$ ), e vice-versa.

Uma conclusão interessante extraída dos comentārios anteriores é a de que o papel passivo de DIII na dinâmica econômica, ao a justar seu crescimento ao dos outros dois setores, torna as caracteristicas desse mesmo crescimento e do próprio nível da participa cão dos salários no produto $\left(w_{3}\right)$ deste setor lexceto quanto à variaça de $\mathrm{w}_{3}$ ) irrelevantes para o comportamento da distribuição glo bal da renda.

De outro lar̉, o fato de os parāmetros $w_{i}$ conterem implicitamente o efeito dos preços relativos - de DIII e de $D_{i}$, o que torna $w_{3}$ um número absoluto, independente dos preços-permite que se reintexprete sua influência na distribuição da renda agregada sob a hipōtese de que estejam ocorrendo modificações nos preços relativos. 
5. CONCLUSOES

Apresentamos os dois principais modelos teóricos de ciclo eco nômico de Kalecki, dos quais o segundo (1954) parece seromais rea lista, como equações a diferenças finitas lineares com coeficientes constantes, e estudamos as condições que originam diferentes trajetōrias temporais. Examinamos à parte a influência que distin tas taxas de crescimento de setores integrados produtores de bens finais (investimento, consumo capitalista e consumo assalariado) $\underline{\text { }}$ xercem sobre a distribuição funcional agregada da renda, dados os parāmetros distributivos a nivel setorial, o que permitiria determinar tendências de alteração na distribuição ao longo do ciclo.

Será desejável que um posterior aprofundamento do tema se pro cesse em pelo menos duas direções:

19) A hipótese de coeficientes constantes pode e deve ser relaxada para incorporar os efeitos semi-exógenos de mudanças tecnológicas, na distribuição de renda, nos padrões de consumo por clas se de renda e nos fatores de risco competitivo e financeiro no transcurso do ciclo. Ao mesmo tempo é necessário aprofundar a análise dos fatores determinantes do componente de tendência, lançando mão em particular das contribuições de Schumpeter na explicação das inovações e tendo em conta os efeitos da prōpria alteração dos parāmetros no curso de cada ciclo - como já sugeria Kalecki (1954), caps. 14 e 15. Esta é, a nosso ver, a integração possível entre ciclo e tendência: a nível propriamente analítico e não teórico, por quanto os princípios explicativos de ambos são distintos.

29) Um modelo de ciclo agregado, como o de Kalecki e todos da mesma "família" (interação multiplicador x função investimento), não consegue captar adequadamente a influência dināmica de distintas configurações técnico-produtivas e competitivas a nível microeconô mico sobre os parãmetros do ciclo. Uma desagregação do modelo, incorporando inclusive os demais componentes semi-exógenos de demanda final (gastos governamentais, saldo de exportações), podcrá convertê-lo em um poderoso instrumental analítico da dinâmica. Cono pximeira aproximação o uso do modelo de 3 setores integrados na análí se do ciclo pode constituir um importante avanço, mas é necessário levar essa desagregação até o nivel de setores ou indústrias, que é a unidade microeconômica de análise pertinente. 


\section{REFERENCIAS}

ALLEN, R.G.D. (1960), Mathematical Economics, 2 a.ed., Macmillan, Londres; trad.esp. Economía Hatemätica, Aguilar, Madri, 1967.

KALECKI, M. (1933), "Outline of a Theory of the Business Cicle", in Studies in the Theony of Business Cycles, A. Ni. Kelley, N. York, 1969; trad.port. "Esboso de uma Teoria do Ciclo Econômico", in Kalecki, M., Cresciment c Ciclo das Economias Capitalistas, citec, São Paulo, 1977 (org. por J.Miglioli).

(1954), Theory of Economic Dynamics, George Allen \& Unwin, Londres; trad.port. Teonia da Dinâmica Economica, Abrii Cultural, São Paulo, 1978 .

(1962), "Observations on the theory of Growth", Economic Journal, vol. LXXII, marso 1962.

(1968a), "The Marxian Equations of Reproduction and Modern Economics", in Social Science Information, 7(6); trad.port. "As Equações Marxistas de Reprodução e a Economia Moderna", in cresciment• e cicl•..., $\bullet$ p.cit.

(1968b), "Trend and the Business Cycle", in Selected Es says on the Dynamics of the Capitalist Economy, Cambridge Uni= versity Press, 1971; trad.port. "Tendência e Ciclo Económico", in Cresciment• e cicl...., op.cit.

PASINETTI, L. (1974), Gnowth and Income Distribution, Cambridge Uni versity Press; trad.port. Cresciment• e Distribüicā॰ de Renda, $Z$ ahar, Rio de Janeiro, 1979.

Possas, M. e BALTAR, P. (1981), "Demanda Efetiva e Dinàmica em Kale cki", Pesquisa e Planejament. Economic•, 11(1), Rio de Janeiro, abril 1981 . 